\title{
Gemelos onfalópagos: reporte de un caso
}

\section{Omphalopagus twins: A case report.}

\author{
Daniela Castro-Maldonado, ${ }^{1}$ María Ameyali Pérez-Huitrón, ${ }^{1}$ Xóchitl Lizbeth Serrano-Almanza, ${ }^{2}$ Bernardo \\ Ramírez-García
}

\begin{abstract}
Resumen
ANTECEDENTES: Los gemelos siameses son casos excepcionales en la práctica médica (incidencia de 1 en 50,000 a 1 en 100,000 nacidos vivos). Los gemelos unidos pueden ser simétricos o asimétricos, cuando un gemelo está completo y sano (gemelo autósito) y el otro es incompleto (gemelo parásito) y depende del autósito para su crecimiento. CASO CLínICO: Gemelos siameses, de dos días de vida extrauterina, intervenidos quirúrgicamente para separar al no viable, por agenesia casi total (únicamente estaba constituido por la región cefálica y tronco dismórfico). El resto de las estructuras del gemelo sano, de género femenino, se observaron sin alteración. El procedimiento se Ilevó a cabo mediante una incisión lateral izquierda y la separación por planos desde la piel, el tejido celular subcutáneo y los trayectos musculares hasta identificar los órganos herniados del gemelo dominante al parásito; ningún órgano vital se encontraba compartido, solo se observó la herniación de una pequeña porción del hígado y de las asas intestinales del gemelo dominante al parásito, por lo que se reintrodujo el sistema digestivo del gemelo dominante y se efectuó la extirpación completa del gemelo parásito. Se cerró la pared con colocación de una malla plana para evitar una posible recidiva de la herniación. El trans y posoperatorio transcurrieron sin complicaciones y con evolución satisfactoria.
\end{abstract}

CONCLUSIONES: La incidencia de gemelos siameses es excepcional. El diagnóstico prenatal se establece por ecografía; sin embargo, la resonancia magnética muestra mejor definición anatómica de las anomalías y permite la planificación obstétrica y los cuidados posnatales adecuados.

PALABRAS CLAVE: Gemelos siameses; gemelos; gemelo parásito; diagnóstico prenatal; cuidados posnatales.

Abstract

BACKGROUND: Siamese twins are rarely in medical practice (incidence is reported as 1 in 50,000 to 1 in 100,000 live births). Twins attached can be symmetrical or asymmetric, when one twin is complete and almost normal (twin self) and the other is incomplete (twin parasite) and depends on the autoto for its growth.

CLINICAL CASE: Siamese twins of two days of extrauterine life, surgically operated to separate the non-viable twin, by almost total agenesis (it was only constituted by the cephalic region and dysmorphic trunk). The rest of the structures of the healthy twin, female, were observed without alteration. The procedure was carried out by means of a left lateral incision and the separation by planes from the skin, the subcutaneous cellular tissue and the muscular paths until identifying the herniated organs of the dominant twin to the parasite; no vital organ was shared, only the herniation of a small portion of the liver and intestinal handles of the dominant twin to the parasite was observed, so the digestive system of the dominant twin was reintroduced and the complete removal of the parasite twin was performed. The wall was closed with placement of a flat mesh to avoid a possible recurrence of herniation. The trans and postoperative period passed without complications and satisfactory evolution.

CONCLUSIONS: The incidence of Siamese twins is exceptional. Prenatal diagnosis is established by ultrasound; however, magnetic resonance imaging shows better anatomical definition of the anomalies and allows obstetric planning and adequate postnatal care KEYWORDS: Siamese twins; Twin; Twin parasite; Prenatal diagnosis; Postnatal care.
${ }^{1}$ Residente de tercer año de Imagenología diagnóstica y terapéutica.

${ }^{2}$ Adscrito al servicio de Imagenología.

Hospital General del Centro Médico Nacional La Raza (Instituto Mexicano del Seguro Social), Ciudad de México.

Recibido: 1 de noviembre 2018

Aceptado: 11 de julio 2019

Correspondencia

María Ameyali Pérez Huitrón

ameyali_ph@hotmail.com

ORCID: 0000-0002-8997-4340

Este artículo debe citarse como Castro-Maldonado D, Pérez-Huitrón MA, Serrano-Almanza XL, RamírezGarcía B. Gemelos onfalópagos: reporte de un caso. Acta Pediatr Mex. 2019;40(5):267-73. 


\section{ANTECEDENTES}

Los gemelos unidos, "siameses", son la consecuencia de alteraciones en los embarazos gemelares monoamnióticos en los que se encuentran dos fetos completamente formados, unidos en diferentes sitios anatómicos. Debido a su baja prevalencia, hasta la fecha no se ha establecido adecuadamente la etiología de esta alteración. ${ }^{1}$

Los gemelos siameses se clasifican de acuerdo con el sitio predominante de unión: toracópagos (tórax), onfalópagos (abdomen), pigópagos (sacro), isquiópagos (pelvis), cranéopagos (cráneo), cefalópagos (cara) o raquípagos (espalda).2,3

En lo que a gemelos asimétricos respecta, se piensa que el de mayor tamaño monopoliza la sangre placentaria y, por tanto, disminuye el aporte en el otro. ${ }^{4}$

Es importante valorar cada caso, con la intención de ofrecer el mejor desenlace obstétrico y perinatal, conociendo la gravedad de la alteración y los órganos que puedan afectarse, para de esta forma disminuir la morbilidad y mortalidad de los pacientes.

A continuación se expone el caso de un gemelo siamés onfalópago asimétrico, que durante el embarazo se estableció el diagnóstico sugerente de gastrosquisis.

\section{CASO CLÍNICO}

Recién nacida de un segundo embarazo, de madre sin antecedentes personales patológicos ni crónico-degenerativos de importancia, con control prenatal adecuado a partir de la octava semana, en quien se sospechó el diagnóstico de gastrosquisis durante el segundo trimestre del embarazo, pero continuó su cursó sin complicaciones (solo tuvo infección de vías urinarias al final del segundo trimestre). En la semana 38 se programó para cesárea; nació una niña sana, sin requerimiento de maniobras adicionales.

La recién nacida obtuvo una calificación de Apgar de 8/9, peso de $4100 \mathrm{~g}$ y talla de $51 \mathrm{~cm}$. A la exploración física se encontró con buen estado general, reactiva, tórax simétrico, con adecuada ventilación y sin dificultad respiratoria; se observó una masa abdominal en la pared anterior, correspondiente a un gemelo siamés, con agenesia casi total, únicamente constituido por la región cefálica y tronco dismórfico. El resto de las estructuras del gemelo sano se observaron sin alteración.

La recién nacida continuó con cuidados generales en la incubadora y se aplazó la lactancia materna; después de dos días de vida extrauterina se realizó una tomografía toraco-abdominal simple y contrastada para establecer el protocolo de estudio y decidir el tipo de procedimiento quirúrgico. Clínicamente se encontraba estable. Figura 1

La tomografía computada en plano sagital mostró ambos gemelos con las siguientes características: gemelo parásito (no viable) y dominante (sana) unidos a través de un defecto en la pared abdominal anterior, de aproximadamente $60 \mathrm{x}$ $58 \mathrm{~mm}$, por el que se evidenció el paso de una porción del lóbulo izquierdo del hígado del gemelo dominante, hacia la rudimentaria cavidad abdominal del gemelo parásito. También se observaron algunas asas del intestino delgado y remanentes de colon proximal proyectados hacia el saco abdominal. La tomografía en 3D mostró la imagen del gemelo dominante formado de acuerdo con su edad; sin embargo, el gemelo parásito fue rudimentario, lográndose visualizar porciones de cráneo, la columna, la pelvis y los huesos proximales de los miembros torácicos y pélvicos; en el tórax se observó el corazón rudimentario, formado por una sola cámara cardíaca. Figura 2 

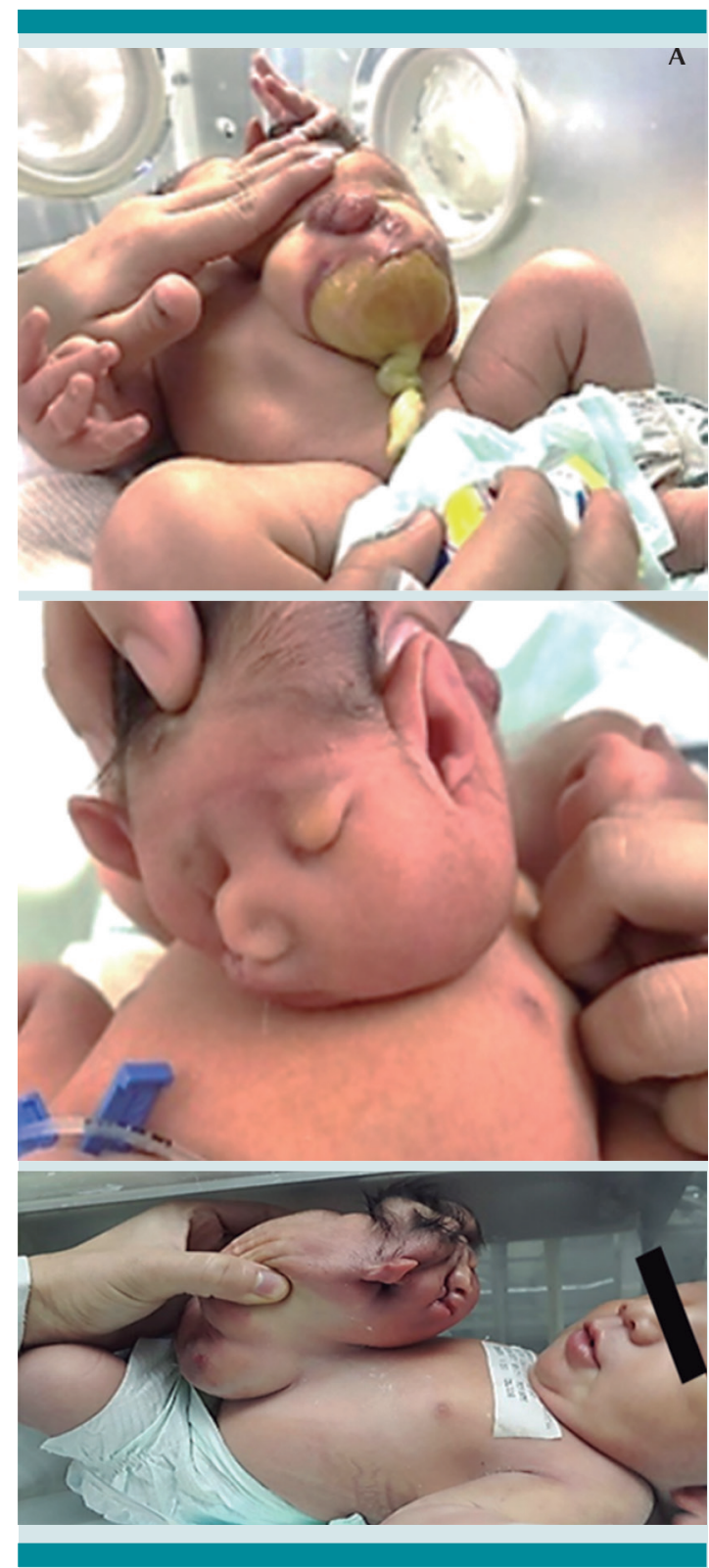

Figura 1. Gemelos siameses recién nacidos. Porción caudal del gemelo parásito (A), tórax del gemelo dominante y encima la porción cefálica del gemelo parásito, con estructuras faciales identificables (ojos, orejas y nariz) (B), y eje longitudinal del paciente y su unión a través de la porción abdominal (C).

Después de tres días de vida extrauterina se practicó la intervención quirúrgica, mediante una incisión lateral izquierda y la separación por planos desde la piel, el tejido celular subcutáneo y los trayectos musculares hasta identificar los órganos herniados del gemelo dominante al parásito; ningún órgano vital se encontraba compartido, solo se observó la herniación de una pequeña porción del hígado y de las asas intestinales del gemelo dominante al parásito, por lo que se reintrodujo el sistema digestivo del gemelo dominante y se efectuó la extirpación completa del gemelo parásito. Se cerró la pared con colocación de una malla plana para evitar una posible recidiva de la herniación. El transoperatorio transcurrió sin complicaciones y la paciente salió del quirófano con intubación orotraqueal.

Aunque la herida quirúrgica fue limpia, permanecía bien afrontada y sin datos de sangrado activo ni infección. Al día siguiente del procedimiento se intentó retirar la intubación orotraqueal; sin embargo, debido a insuficiencia respiratoria permaneció intubada 5 días más, hasta que toleró la extubación de forma adecuada. Además, requirió apoyo inotrópico con dobutamina por tensión arterial media por debajo del percentil 5, con suspensión del protocolo cuatro días después, con evolución satisfactoria.

La recién nacida continuó con nutrición parenteral durante una semana, hasta que toleró la vía oral. Después de descartar la coexistencia de posibles anomalías congénitas asociadas, fue dada de alta del hospital a los 17 días de vida extrauterina, hemodinámicamente estable, sin desequilibrio hidroelectrolítico y neurológicamente sin datos de focalización.

La paciente se programó a citas de control en el servicio de Cirugía pediátrica; puesto que ya no acudió se desconoce su estado de salud actual. 


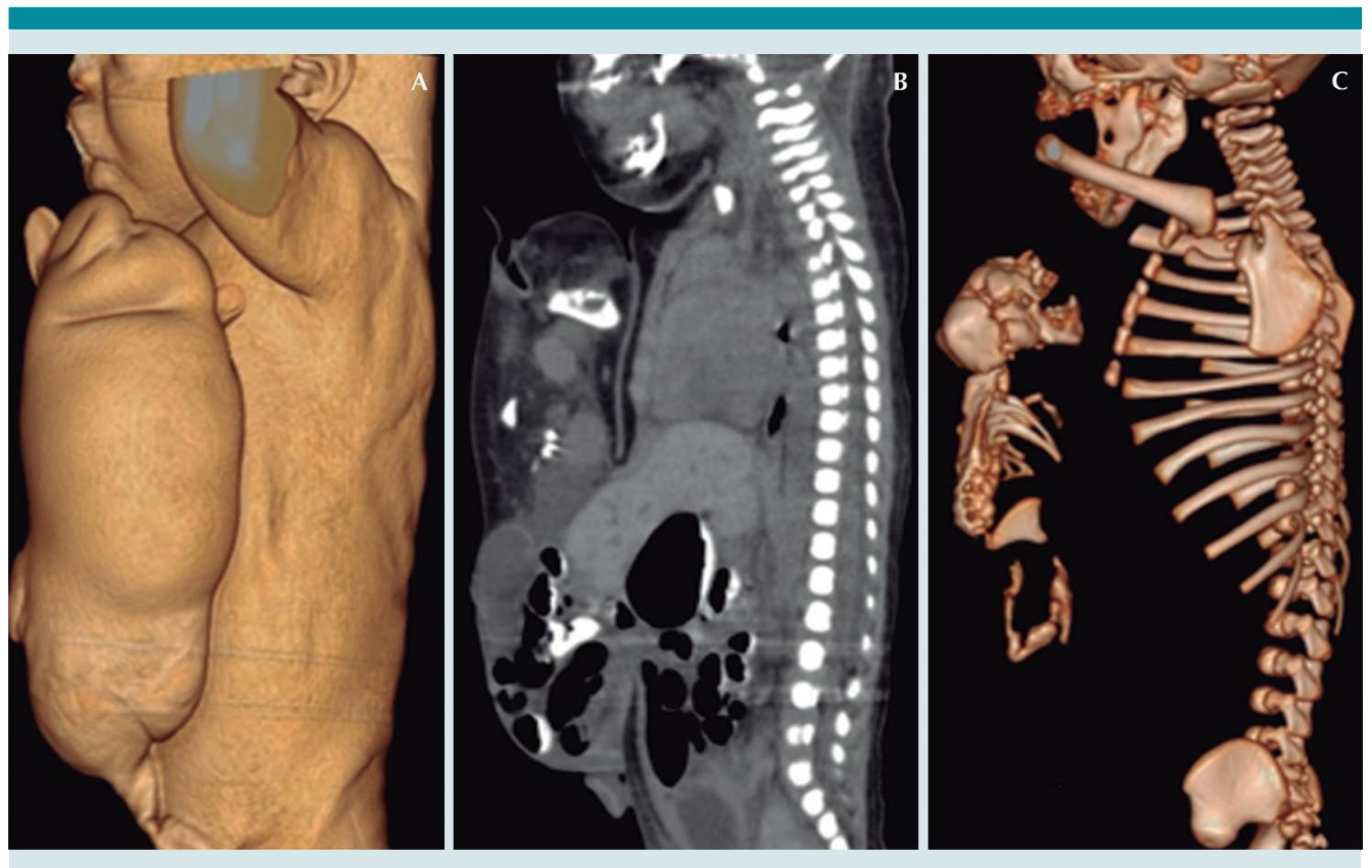

Figura 2. Tomografía en 3D. Reconstrucción volumétrica que muestra la relación entre ambos gemelos (A,B); órganos herniados a través de la pared abdominal (lóbulo izquierdo del hígado e intestino delgado), comprobado con medio de contraste positivo vía oral; y estructuras óseas de ambos gemelos, independientes (C).

\section{DISCUSIÓN}

La incidencia de gemelos siameses asimétricos es de 0.1 en 100 a 200 mil casos, con una tasa de supervivencia del gemelo dominante más alta que la del gemelo siamés simétrico. ${ }^{5}$

Hasta la fecha no se han establecido las causas exactas de la alteración de gemelos siameses; ${ }^{6}$ sin embargo, la teoría más aceptada establece posibles alteraciones en la fusión completa de la masa celular interna, que origina distintos tipos de gemelos unidos, lo que resulta en la fisión de órganos provocada por defectos en la blastogénesis. ${ }^{4}$ La teoría de la fusión sugiere que la división ocurre después del decimotercer día, con la completa separación de los primeros blastómeros, seguida de la fusión parcial. En ese momento existe la posibilidad de que un gemelo se adhiera (parásito) y el otro se desarrolle normalmente (autósito). ${ }^{7}$

Algunos autores señalan que en los gemelos asimétricos, uno recibe mayor cantidad de sangre placentaria, lo que causa el deterioro del otro. ${ }^{4}$ Spencer R, citado por Gómez-Cadena y su grupo, ${ }^{1}$ propuso una clasificación para los gemelos siameses de acuerdo con el sitio predominante de unión: toracópagos (tórax), onfalópagos (abdomen), pigópagos (sacro), isquiópagos (pelvis), craneópagos (cráneo), cefalópagos (cara) o raquípagos (espalda). ${ }^{1-4}$ Otra forma de clasificación es 
mediante simetría o asimetría. En la primera se encuentran los gemelos con desarrollo y tamaño similar, y en la segunda los gemelos unidos asimétricos, también Ilamados heterópagos, ${ }^{4}$ cuando uno de ellos (gemelo autósito o dominante) es completo y relativamente sano y el otro (gemelo parásito) es incompleto, unido al autósito y depende de él para su crecimiento. ${ }^{8}$

El gemelo parásito puede, a su vez, designarse endoparásito o fetus in fetu cuando se encuentra dentro del gemelo dominante, o ectoparásito cuando sólo está unido a la superficie de éste. De la Torre-Mondragón y su grupo ${ }^{8}$ recomiendan que para nombrarlos, primero debe especificarse que son gemelos unidos asimétricos, y después señalar el sitio de unión y las estructuras anatómicas que forman el parásito. Por tanto, siguiendo esta nomenclatura, nuestro caso correspondió a un gemelo unido asimétrico onfalópago céfalo-toraco-isquion.

Cuando el gemelo es endoparásito debe descartarse la coexistencia de teratoma y fetus in fetu; para ello es importante diferenciarlos por algunas características, por ejemplo: si mantiene un saco distinto, está cubierto de piel sana, de forma parcial o completa; contiene partes anatómicas reconocibles, se mantiene unido al huésped por pocos vasos sanguíneos o comparten un sitio anatómico en común. ${ }^{3}$

Los gemelos unidos ectoparásitos reportados en la bibliografía médica son poco frecuentes y la mayoría están formados por estructuras de la mitad inferior del cuerpo; por tanto, nuestro caso resulta interesante, puesto que los gemelos siameses asimétricos se encontraban unidos por la parte superior del abdomen. ${ }^{8}$

El diagnóstico clínico es difícil de establecer. En estos casos, la evaluación ultrasonográfica prenatal es un estudio importante, como ocurrió en nuestra paciente, por la sospecha durante el embarazo de gastrosquisis, hasta evidenciar los gemelos al momento del parto; no obstante, ante la duda diagnóstica puede indicarse, incluso, resonancia magnética para una mejor caracterización. ${ }^{1,2,9}$

El estudio de elección para el diagnóstico prenatal es la resonancia magnética, pues puede evaluar de mejor forma las estructuras anatómicas, y ofrece buena interfase entre los tejidos y mejor campo de visión, sobre todo en el tercer trimestre, cuando el feto es más grande y tiene menor movimiento. La resonancia magnética es útil cuando los hallazgos ecográficos del feto son ambiguos o inciertos. ${ }^{10}$

Después del nacimiento puede evaluarse a los gemelos mediante diversos métodos; por ejemplo, el estudio contrastado permite una evaluación completa de los conductos gastrointestinal y urogenital, y la angiografía para definir la vascularidad; todos son útiles para la planeación quirúrgica. ${ }^{4}$ El ecocardiograma es importante porque ha encontrado una alta incidencia de malformaciones congénitas cardiacas en los autósitos. ${ }^{6,11}$

Aunque el diagnóstico de gemelos unidos representa un reto para el cirujano pediatra, debido a la gran diversidad y complejidad de muchos casos, en los gemelos siameses simétricos debe lograrse la separación y supervivencia de ambos; ${ }^{11}$ comparados con los asimétricos, donde la finalidad es la supervivencia del gemelo dominante, con las menores secuelas. ${ }^{8}$ La paciente de este estudio fue dada de alta del hospital sin complicaciones; por tanto, superó las expectativas de vida, ya que generalmente fallecen horas después de vida extrauterina o posterior a la intervención quirúrgica.

En la bibliografía nacional existen algunos casos reportados (Cuadro 1) que si bien son pocos, representan un referente en el conocimiento de la alteración. ${ }^{12-17}$ Por tanto, es importante reportar este tipo de casos, ya que además suponen pacientes dados de alta y sin complicaciones aparentes. 
Cuadro 1. Serie de casos reportados en la bibliografía nacional

\begin{tabular}{|c|c|c|c|c|c|c|}
\hline $\begin{array}{l}\text { Autor } \\
\text { (año de publicación) }\end{array}$ & $\begin{array}{c}\text { Entidad } \\
\text { federativa }\end{array}$ & $\begin{array}{l}\text { Tipo de } \\
\text { unión }\end{array}$ & $\begin{array}{l}\text { Simétricos o } \\
\text { asimétricos }\end{array}$ & $\begin{array}{c}\text { Clasificación morfológica } \\
\text { de Spencer }\end{array}$ & Sexo & $\begin{array}{l}\text { Último reporte de } \\
\text { salud conocido* }\end{array}$ \\
\hline $\begin{array}{l}\text { Hernández-Valencia M, } \\
\text { et al (1998). } .^{12}\end{array}$ & $\begin{array}{l}\text { Estado de } \\
\text { México }\end{array}$ & Ventral & Asimétricos & Cefalotoracoonfalópagos & $\mathrm{F}$ & Falleció \\
\hline $\begin{array}{l}\text { De la Torre-Mondragón L, } \\
\text { et al (2000). }{ }^{8}\end{array}$ & $\begin{array}{l}\text { Ciudad de } \\
\text { México }\end{array}$ & Ventral & Asimétricos & Isquiopagos hemicorporis & M & $\begin{array}{c}\text { Egresó y permaneció } \\
\text { en seguimiento } \\
\text { durante un año. }\end{array}$ \\
\hline $\begin{array}{l}\text { García-Chávez M, et al } \\
\text { (2010). }{ }^{7}\end{array}$ & Tabasco & Lateral & Asimétricos & Parapagos dicéfalos & $\mathrm{F}$ & Falleció \\
\hline $\begin{array}{l}\text { Panduro-Barón G, et al } \\
(2013) .^{13}\end{array}$ & Jalisco & Ventral & Asimétricos & $\begin{array}{c}\text { Toracoonfalópagos } \\
\text { dicéfalos }\end{array}$ & $\mathrm{D}$ & Falleció \\
\hline $\begin{array}{l}\text { Panduro-Barón G, et al } \\
\text { (2013). }{ }^{13}\end{array}$ & Jalisco & Ventral & Asimétricos & $\begin{array}{l}\text { Toracoonfalópagos } \\
\text { dicéfalos }\end{array}$ & $\mathrm{D}$ & Falleció \\
\hline $\begin{array}{l}\text { Panduro-Barón G, et al } \\
\text { (2013). }{ }^{13}\end{array}$ & Jalisco & Ventral & Simétricos & Toracópagos dicéfalos & $\mathrm{D}$ & Falleció \\
\hline $\begin{array}{l}\text { González-Cortés BGl, et } \\
\text { al (2015). }{ }^{14}\end{array}$ & $\begin{array}{l}\text { Estado de } \\
\text { México }\end{array}$ & Ventral & Asimétricos & $\begin{array}{l}\text { Toracoonfalópagos } \\
\text { dicéfalos }\end{array}$ & M & Falleció \\
\hline $\begin{array}{l}\text { Gómez-Murillo SY, et al } \\
(2016) .^{15}\end{array}$ & $\begin{array}{l}\text { Ciudad de } \\
\text { México }\end{array}$ & Ventral & Simétricos & $\begin{array}{l}\text { Toracoonfalópagos } \\
\text { dicéfalos }\end{array}$ & M & $\begin{array}{l}\text { Uno de ellos } \\
\text { sobrevivió } \\
\text { seis meses. }\end{array}$ \\
\hline $\begin{array}{l}\text { Arteaga-Yañez J, et al } \\
(2016) \cdot{ }^{10}\end{array}$ & Sinaloa & Ventral & Asimétricos & $\begin{array}{l}\text { Toracoonfalópagos } \\
\text { dicéfalos }\end{array}$ & M & Falleció \\
\hline $\begin{array}{l}\text { Gómez-Cadena J, et al } \\
\text { (2018). }{ }^{16}\end{array}$ & $\begin{array}{l}\text { Ciudad de } \\
\text { México }\end{array}$ & Lateral & Asimétricos & Parapagos dicéfalos & $\mathrm{F}$ & Falleció \\
\hline $\begin{array}{l}\text { Gómez-Cadena J, et al } \\
\text { (2018). }{ }^{16}\end{array}$ & $\begin{array}{l}\text { Ciudad de } \\
\text { México }\end{array}$ & Ventral & Asimétricos & Toracoonfalópagos & M & Falleció \\
\hline $\begin{array}{l}\text { Gómez-Cadena J, et al } \\
\text { (2018). }{ }^{16}\end{array}$ & $\begin{array}{l}\text { Ciudad de } \\
\text { México }\end{array}$ & Ventral & Asimétricos & $\begin{array}{l}\text { Toracoonfalópagos } \\
\text { dicéfalos }\end{array}$ & M & Falleció \\
\hline $\begin{array}{l}\text { Gómez-Cadena J, et al } \\
\text { (2018). }{ }^{16}\end{array}$ & $\begin{array}{l}\text { Ciudad de } \\
\text { México }\end{array}$ & Ventral & Asimétricos & Cefalotoracoonfalópagos & $\mathrm{F}$ & Falleció \\
\hline $\begin{array}{l}\text { Gómez-Cadena J, et al } \\
\text { (2018). }{ }^{16}\end{array}$ & $\begin{array}{l}\text { Ciudad de } \\
\text { México }\end{array}$ & Lateral & Asimétricos & Parapagos dicéfalos & M & Falleció \\
\hline $\begin{array}{l}\text { Nava-Martínez M, et al } \\
(2018) .{ }^{17}\end{array}$ & $\begin{array}{l}\text { Ciudad de } \\
\text { México }\end{array}$ & Dorsal & Simétricos & Pigópagos & M & Ambos egresaron. \\
\hline $\begin{array}{l}\text { Castro-Maldonado D, et } \\
\text { al (2019). Caso actual }\end{array}$ & $\begin{array}{l}\text { Ciudad de } \\
\text { México }\end{array}$ & Ventral & Asimétricos & Onfalópagos & $\mathrm{F}$ & Egresó. \\
\hline
\end{tabular}

* Pudo implicar pérdida durante el seguimiento.

\section{CONCLUSIONES}

La incidencia de gemelos siameses es poco frecuente, sin predisposición genética; la forma heterópaga, en la que un feto no está completamente desarrollado, representa un caso excepcional. El diagnóstico prenatal se establece por ecografía; sin embargo, la resonancia magné- tica muestra mejor definición anatómica de las anomalías y permite la planificación obstétrica y los cuidados posnatales adecuados.

\section{Agradecimientos}

A Raquel Mejía Kato por la adecuación de las imágenes. 


\section{REFERENCIAS}

1. Singh $\mathrm{Y}$, et al. Early prenatal diagnosis in a case of conjoined twin with Craniopagus and Thoraco-omphalopagus. Med J Armed Forces India. 2012;68(4):381-382. doi:10.1016/j. mjafi.2011.12.005

2. Sewell EK, et al. Impact of prenatal diagnosis of fetus-in-fetu. J Neonatal Perinatal Med. 2017;10(3):333-338.

3. Chelliah KK, et al. Multimodality Imaging in the Assessment of thoraco-omphalopagus conjoined twin: Lessons to learn. Case Rep Radiol 2012;2012:1-4. DOI: $10.1155 / 2012 / 564036$

4. ain PK, Budhwani KS, Gambhir A, Ghritlaharey R. Omphalopagus Parasite: A Rare Congenital Anomaly. J Pediatr Surg. 1998;33(6):946-947. DOI: https://doi.org/10.1016/ S0022-3468(98)90682-0\%0A.

5. Sharma G, Mobin SSN, Lypka M, Urata M. Heteropagus (parasitic) twins: A review. J Pediatr Surg. 2010;45(12):24542463. DOI: 10.1016/j.jpedsurg.2010.07.002

6. Das S, Ghosh D, Biswas S, et al. Heteropagus Twins-A Tale of Two Cases. Indian J Surg. 2011;73(3):217-220. DOI: 10.1007/s12262-010-0228-8

7. García-Chávez M, et al. Gemelos unidos parápagos dicéfalos. Reporte de un caso y revisión de la literatura. Rev Mex Pediatr 2010;77(2):59-63. https://scielo.conicyt.cl/ $\mathrm{pdf} / \mathrm{rcp} / \mathrm{v84n6/art09.pdf}$.

8. De la Torre L, et al. Gemelos unidos asimétricos. Ischiopygopagus, hemicorporis total izquierdo. Acta Pediátr Méx 2000;21(1):3-7.
9. Mathew RP, et al. Conjoined twins - role of imaging and recent advances. J Ultrason. 2017;17(71):259-266. DOI: 10.15557/jou.2017.0038

10. Arteaga-Yañez J, et al. Siameses toracópagos Conjoined thoracopagus twins: a case. An Radiol Méx 2016;15(3):244247.

11. Dejene B, et al. Heteropagus (parasitic) twins. J Pediatr Surg Case Reports. 2018;37:44-49. DOI: 10.1016/j. epsc.2018.07.019

12. Hernández-Valencia M, et al. Embarazo Janiceps, cefalotoracoabdominopago. Ginec Obs Mex 1998;66:499-502.

13. Panduro-Barón G, et al. Gemelos unidos (siameses). Reporte de tres casos. Rev Médica MD 2013;4(4):277-279. http://www.medigraphic.com/cgi-bin/new/resumen. cgi?IDARTICULO=44044.

14. Cortés BGIG, et al. Siameses toracópagos: presentación de caso y revisión de la literatura. Perinatol y Reprod Humana. 2015;29(3):130-133. DOI: 10.1016/j. rprh.2015.12.006

15. Gómez-Murillo SY, et al. Siameses onfalópagos: presentación de un caso. Acta Pediátr Méx 2016;35(6):469. DOI: 10.18233/apm35no6pp469-476

16. Gómez-Cadena J, et al. Gemelos unidos (siameses): descripción de hallazgos anatomopatológicos. Ginec Obs Mex. 2018;86(12):823-830. DOI: https://doi.org/10.24245/gom. v86i12.2449

17. Nava-Martínez M, et al. Manejo anestésico durante la separación orgánica total de gemelos unidos pigópagos. Rev Med Inst Mex Seguro Soc 2018;56(1):106-111. 